\title{
LAS LECCIONES DE TERSITES. SEMBLANZA DE UNA VIDA Y UNA ÉPOCA, 1951-2016
}

por Raimundo Cuesta Fernández, Madrid: Visión Libros, 2017, 350 páginas. ISBN: 978-84-9011-630-2.

\section{A LA SOMBRA DEL AUTOR}

El autor y su sombra figura como el encabezamiento que cobija las tres partes (ensayo introspectivo, entrevista y reseña) que hemos elaborado a cuatro manos entre Raimundo Cuesta y quien escribe esta glosa final. Sin duda, tal título representa en cierta manera un guiño de complicidad con El caminante y su sombra (1789), obra de Friedrich Nietzsche en la que el errabundo filósofo dialoga con ese otro yo que le daba compañía en sus largas jornadas de caminante solitario y pensante. Salvando todas las distancias, se me ocurre que yo mismo escribo esta reseña a la sombra de una figura que, como se muestra en Las lecciones de Tersites, dispone de una batería de recursos, así como de una historia de vida, muy difíciles de resumir en una breve reflexión crítica. Por ello me centraré mejor en las cuestiones personales y subjetivas en las que, a mi modo de entender, se basa mi relación intelectual con el autor, Raimundo Cuesta. En primer lugar, como visión de conjunto, la primera dificultad que se me presenta en esta tarea, es la de valorar su ingente producción intelectual, contraponerla a las dificultades a las que nos enfrentamos los jóvenes historiadores en la actualidad. En su libro afloran las lecciones tanto de un maestro (entendido como persona que enseña un arte $\mathrm{u}$ oficio, en este caso, el de profesor de la enseñanza secundaria) como de un brillante historiador de la educación y, más recientemente, las de un excelente crítico de la cultura con el que coincido en muchos puntos de vista. En este caso los elogios no se tratan solamente de un formalismo. Me remito a las webs de Fedicaria y de Nebraskaria.

Raimundo Cuesta nos presenta en esta ocasión una interesante introspección personal sobre su vida y sobre su trabajo, muy en la línea 
de las nuevas formas de narrativa postmoderna, entre las cuales, Jaume Aurell y Peter Burke señalan la aparición de un nuevo género autobiográfico que constituye una auténtica "tendencia intelectual». ${ }^{1}$ Esta nueva narrativa biográfica es distinta de la tradicional, presenta algunas características diferenciadoras. En ella se comienza a abandonar cualquier pretensión de objetividad, especialmente en lo que se refiere a la descripción del yo, constituyéndose, tal y como lo definíamos en la entrevista parafraseando a Aitor Bolaños de Miguel, en un auténtico «experimento postmoderno». Una manera de «subrayar la posibilidad de otras formas de representar el pasado con la finalidad de proyectar nuevas maneras de lidiar con el tiempo.» ${ }^{2} \mathrm{Si}$ el conocimiento social, esto es, las ciencias sociales, son subjetivas, tal y como ya enuncian autores emergentes como César Rendueles, nuestra propia cotidianeidad también lo es. ${ }^{3}$ Fue Raymond Williams, entre otros, quien consideró la experiencia (experiencia vivida) como una mediación fundamental para la conformación de la subjetividad de los sujetos. Describía más concretamente este autor el «verdadero proceso social» como «una experiencia histórica activa y consciente así como, por descuido, una experiencia histórica pasiva y objetivada». ${ }^{4}$ De modo que, este relato sobre la vida de Tersites tiene valor también como una fuente de información tanto para la historia de la educación como para la propia historia contemporánea o historia reciente de nuestro país.

Sin embargo, aquí y ahora en mi escrito me interesa más que nada subrayar, reconstruir y trazar el itinerario de mi relación intelectual y personal con el autor de Las lecciones de Tersites. Nuestros caminos se cruzaron con motivo de sus clases en el Máster de Educación Secundaria durante el curso transcurrido entre 2010 y 2011; allí comencé a ponerme en contacto con esto de la «didáctica crítica de las ciencias sociales». Pero las cuestiones relativas a la temática del Máster citado no fueron las pri-

\footnotetext{
${ }^{1}$ Jaume Aurell y Peter Burke, «Las tendencias recientes: del giro lingüístico a las historias alternativas» en Comprender el pasado. Una historia de la escritura y el pensamiento histórico, ed. Jaume Aurell et al. (Madrid: Akal, 2013), 287-339 (cita en 308).

2 Aitor M. Bolaños de Miguel, «Experimentos historiográficos postmodernos», Revista de historiografía, 13 (2010): 100-114 (cita en 101).

${ }^{3}$ Afirma el sociólogo César Rendueles que «Las ciencias sociales son elaboraciones refinadas de nuestras prácticas cognitivas rutinarias. Son praxiologías, es decir, saberes cotidianos, no científicos». César Rendueles, En bruto. Una defensa del materialismo histórico (Madrid: La Catarata, 2016), 66.

${ }^{4}$ Raymond Williams, Marxismo y literatura (Barcelona: Península, 1997), 107.
} 
meras que nos unieron. Nos unió una concepción similar, no dogmática, de la cultura como herramienta de impugnación del sistema capitalista y de liberación de los individuos en las sociedades del capitalismo tardío. He de confesar que a mí me sorprendió enormemente la predisposición de aquel profesor hasta entonces desconocido - a pesar de que yo cursé Bachillerato en el mismo instituto en el que él ejerció la docencia, donde no coincidimos - a participar en el ambiente estudiantil creado en torno a las plataformas anti-Bolonia, que estallaron en una protesta estudiantil en el año 2007 contra las reformas universitarias de corte neoliberal que se estaban llevando a cabo y hoy por desgracia ya asentadas. Por aquel entonces yo militaba en una organización política juvenil y durante los años de licenciatura había observado desconsolado lo que a mí me parecía una falta de compromiso cívico por parte del profesorado universitario, el cual hoy, como venganza retroactiva, sufre las consecuencias del sistema burocrático que se consolidaba durante el gobierno de José Luis Rodríguez Zapatero y que solo los estudiantes de aquel entonces nos atrevimos a criticar públicamente, puesto que no creo que no fuésemos los únicos en vislumbrar sus contradicciones. ${ }^{5} \mathrm{Y}$ dentro de esas coordenadas, preparamos una charla sobre las dimensiones de la Transición desde un punto de vista que nada tiene que ver con la noción de una "Transición modélica». Era el primer semestre del curso académico 2010-2011, un poco antes de que desde los movimientos sociales configurados en torno al $15 \mathrm{M}$, en 2011, se comenzase la construcción de relatos alternativos sobre el tema, ciertamente contrahegemónicos. ${ }^{6}$

Solo con el tiempo supe comprender la veta más profunda del pensamiento de Raimundo Cuesta, perceptible ya desde la fundación del Grupo Cronos con el que ganó en 1984 el primer premio nacional «Francisco Giner de los Ríos» a la innovación educativa. ${ }^{7}$ Desde los años noventa hasta ahora destaca su dedicación a la Federación Icaria (Fedicaria) en

\footnotetext{
${ }^{5} \mathrm{Al}$ respecto, pueden consultarse mis intervenciones en el claustro y consejo de gobierno de la Universidad de Salamanca, así como la participación en algunos de los debates planteados en la televisión de dicha Universidad.

${ }^{6}$ En esta línea, véase la propuesta de Juan Andrade, El PCE y el PSOE en (la) Transición (Siglo xxI: Madrid, 2012). En la intervención de Raimundo Cuesta se criticaba abiertamente la posición de obras como la de Salvador Sánchez-Terán, La transición: síntesis y claves (Barcelona: Planeta, 2008).

${ }^{7}$ Raimundo Cuesta y Manuel Fernández Cuadrado, «El proyecto Cronos para la enseñanza de las Ciencias Sociales: balance y perspectivas de un itinerario pedagógico», Aspectos críticos de las Ciencias Sociales, 14 (2000): 99-137.
} 
la que recientemente se me ha invitado a participar como miembro del consejo editorial de su publicación Con-Ciencia Social, proyecto al que acudo con voluntad de seguir aprendiendo. Es sin duda el núcleo de investigadores y de investigadoras conformado en torno a Fedicaria y la publicación de la revista Con-Ciencia Social el lugar al que se puede acudir para encontrar numerosas sugerencias acerca de estos planteamientos. Esta revista ahora inicia el camino de su segunda etapa, orientada a la crítica de la cultura en un sentido más amplio aunque manteniendo el universo de las preocupaciones que ha ido desarrollando el grupo a lo largo de los últimos años. ${ }^{8}$ Aprendí que desde Fedicaria se entienden las instituciones escolares como aparatos de control de cuerpos y, sobre todo, más recientemente, de subjetividades. En lo que Raimundo Cuesta ha definido como una «sociedad medusa», la escuela serviría para el control biopolítico de los sujetos, ese control que no necesita - que cada vez necesita menos- de la violencia física para desarrollarse, incardinándose en el propio deseo de los individuos. Nótense en estas afirmaciones las influencias foucaultianas del autor, también desarrolladas por autores como Michael Hardt o Antonio Negri, entre otros. La otra gran influencia que debe destacarse es la obra del sociólogo francés Pierre Bourdieu. Las instituciones escolares se habrían convertido en un elemento más, quizá uno de los más importantes, de los que dispone el poder para generar sociedades dóciles en las que la reproducción de las desigualdades, ya sean con carácter de clase, de género, etc. se naturalizan, desechándose cualquier consideración crítica sobre la generación (sociogénesis) de las mismas. Y precisamente sobre lecturas compartidas de estos y otros autores pude ir construyendo el aparato teórico de mi tesis doctoral, en la que trato sobre el surgimiento de estructuras de poder vinculadas al origen del Estado moderno durante la temprana Edad Moderna como son las Universidades, así como algunos fenómenos de resistencia de estas corporaciones frente al poder monárquico. Está por ver si el crudo panorama laboral al que nos enfrentamos los jóvenes investigadores en la actualidad me vaya a permitir continuar desarrollando esta línea de investigación. En este sentido, una diferencia clara que separa a mi generación de la suya es el hecho de vivir en un periodo de regresión de los

\footnotetext{
${ }^{8}$ Algunas referencias destacables: Raimundo Cuesta, "Genealogía y cambio conceptual. Educación, historia y memoria», Archivos Analíticos de Políticas Educativas, 22 (23), (2014): 1-25; Raimundo Cuesta y Juan Mainer, «Guardianes de la tradición y esclavos de la rutina: historia del campo profesional de los catedráticos de instituto», Historia y Memoria de la Educación, 1 (2014): 351-393.
} 
derechos básicos conquistados por la clase trabajadora, así como la incertidumbre y el desasosiego que generan la inexistencia de alternativas políticas capaces de revertir este proceso y que configuran algunas de las claves de las sociedades postmodernas.

Coincidíamos, por tanto, en nuestra crítica de la escuela y de las instituciones escolares como elemento indisociable de la historia del capitalismo, y de su génesis durante la temprana Edad Moderna, para poder comprender su evolución hasta el presente, tal y como muestra Cuesta en Felices y escolarizados: crítica de la escuela en la era del capitalismo (2005). La lectura de esta obra me abrió la puerta a una especie de despertar intelectual en el que se me permitiría como investigador, contribuir en mi crítica al capitalismo, de una forma abierta, no dogmática, pero suficientemente seria para ser tenida en cuenta. Algo que no había aprendido en la licenciatura y que poco o nada practican muchos de mis colegas, jóvenes investigadores e investigadoras que, como yo, comenzamos nuestra andadura en este oficio de (re)escribir, (re)pensar y (re)interpretar la historia. El modo de educación que Raimundo Cuesta define como «tecnocrático de masas», implantado en nuestro país en el tardofranquismo, se habría mantenido hasta la actualidad, siendo la denuncia que establecíamos desde las plataformas anti-Bolonia la culminación de un proceso que debe ser comprendido mejor en el tiempo largo para sacarle todo su jugo. De modo que los efectos de la globalización, mercantilización y psicopedagogización del conocimiento, se podían poner en relación con una red ya instalada de nudos obligatorios de reagrupamiento de mensajes al servicio de una ortopedia social suave, persuasiva y devastadora que nos situarían ante un futuro de carácter distópico cuyas consecuencias ya comenzamos a sufrir. ${ }^{9}$

Frente a este panorama, quizá un tanto desalentador, desde Fedicaria se plantea también una alternativa, codificada en el programa de la «didáctica crítica de las ciencias sociales», definida como actividad teórica y práctica que aboga por una educación histórica del deseo o de una relación dialéctica de este con las condiciones sociohistóricas (las necesidades) y (el deseo de) los agentes educativos. De ahí se infiere una concepción de la práctica pedagógica como un tipo de relación social inscrita en

\footnotetext{
${ }^{9}$ Raimundo Cuesta. «La escolarización de masas: un sospechoso y «feliz» consenso transcultural», Cuadernos de Pedagogía, 334 (2004): 81-85.
} 
las relaciones de poder operantes en la escuela, fundándose -la didáctica crítica- en torno a tres vectores: «la crítica de la cultura, el análisis genealógico y una forma alternativa de política de la cultura». ${ }^{10}$ Cinco postulados, a modo de enunciados-guía de esta práctica, serían: problematizar el presente, pensar históricamente, educar el deseo, aprender dialogando, impugnar los códigos pedagógicos y profesionales. ${ }^{11}$ ¡Cómo no íbamos a estar de acuerdo! En efecto, en esta didáctica el concepto crítico está relacionado con pensar contra lo establecido, afirmar que lo que existe no nos gusta, y se mantiene la esperanza de la posibilidad de conseguir una alfabetización alternativa de los sujetos, de algún modo aquello que reclamábamos en dichas asambleas que tantas horas se llevaron de nuestras licenciaturas. Frente al triunfo de la razón tecnocrática, la didáctica crítica se erige como una forma de pensar y de actuar de carácter contra-hegemónico. Después de todo, continúa existiendo un principio de esperanza a pesar del desarrollo de los dispositivos de control de orden neoliberal. Las preguntas que hoy me hago son las siguientes: ¿Por qué ningún profesor hasta entonces nos lo había explicado de manera tan clara? ¿Qué temían?

Se sitúa esta propuesta, también, en el gramsciano terreno de la hegemonía, un concepto hoy en boga pero hacia el que Raimundo Cuesta ya apuntaba en su obra Los deberes de la memoria en la educación (2007). En ella se proponía - emulando a Walter Benjamin en sus Tesis de filosofía de la historia (1940)_ cepillar la historia a contrapelo. Según esta obra, el historiador crítico debería abandonar la complicidad afectiva, la empatía del historiador historicista, del intelectual acrítico con el pasado comprendido como la narración y rememoración contemplativa de las gestas de los vencedores. ${ }^{12}$ Era lo que yo pensaba pero que hasta el momento no había sabido formular. Y, por otra parte, siguiendo a Habermas, se plantea unos usos públicos de la historia en la escuela. Estos usos públicos pudieran ser - de ser tenidos en cuenta - el antídoto para encontrar una dimensión útil o práctica, en un estadio de neoliberalismo educativo, no solo para revertir valores tan negativos como los que este plantea en la

${ }^{10}$ Raimundo Cuesta et al., «Didáctica crítica. Allí donde se encuentran la necesidad y el deseo», Con-Ciencia Social, 9 (2005): 17-54 (cita en 20).

${ }^{11}$ Raimundo Cuesta, «La educación histórica del deseo. La didáctica de la crítica y el futuro del viaje a Fedicaria», Con-Ciencia Social, 3 (1999): 80-91.

${ }^{12}$ Raimundo Cuesta, Los deberes de la memoria en la educación (Barcelona: Octaedro, 2007), 50. 
educación, sino de las propias ciencias sociales, las cuales poco a poco van desapareciendo de los programas educativos. Y de nuevo, otro punto de encuentro, puesto que fruto de esta disputa en torno a la utilización de la figura del filósofo italiano Antonio Gramsci después del movimiento del $15 \mathrm{M}$ y su institucionalización a través de algunos partidos políticos, un grupo de personas (jóvenes estudiantes e investigadores) decidimos poner en marcha el Grupo de Estudios Culturales Antonio Gramsci. La idea era contribuir a un pensamiento de carácter contrahegemónico y decididamente anticapitalista, antifascista y feminista; sin medias tintas, puesto que la reforma (o contrarreforma) a la que nos enfrentamos no las tiene.

Finalmente, y ya colaborando de manera activa de forma intergeneracional y, por cierto, muy rica desde el punto de vista intelectual, los compañeros del Antonio Gramsci, junto con los miembros de Fedicaria, vinimos a presentar, entre otras actividades, La venganza de la memoria $y$ las paradojas de la historia (2015), donde se plantea una propuesta epistemológica y metodológica más clara. Se destaca en este ensayo la norma historiográfica dominante como memoria del poder, como un discurso legitimador de las distintas formas de dominación. Frente a este tipo de mirada se apuesta por una historia como método de crítica (o actividad crítica) de la razón moderna. ${ }^{13}$ También pudimos disfrutar de la realización de seminarios para preparar un ensayo historiográfico que ha supuesto mi primer libro, y que sin esta colaboración recíproca y colectiva habría quedado mucho más deslucido. Comentaré finalmente que una de las cuestiones a destacar dentro de estas colaboraciones es el carácter igualitario que establecíamos en nuestra relación intelectual, es decir, que todos y todas somos capaces de aprender de los y las demás. Esta horizontalidad de posiciones de poder contribuye a enhebrar una nueva forma de sociabilidad, no solo de carácter académico, sino también personal, muy enriquecedora.

Gustavo Hernández Sánchez

Universidad de Salamanca gustavohistoria@usal.es

\footnotetext{
${ }^{13}$ Gustavo Hernández, «Historia con Memoria (y Educación)», Historiografías, 10 (2015): 150-156.
} 